\section{neuro- psychiatrie}

vereinigt mit psychiatrie \& psychotherapie

\title{
Bericht aus dem Vorstand der ÖGKJP
}

\author{
Andreas Karwautz
}

(c) Springer-Verlag GmbH Austria, ein Teil von Springer Nature 2019

\section{Sehr geehrte Kolleginnen und Kollegen,}

diesmal kann ich berichten, dass uns für den 18. ESCAP-Kongress, der vom 30. Juni bis 2. Juli 2019 im Kongresszentrum Hofburg in Wien stattfinden wird, eine Rekordanzahl von Einreichungen von über 1000 Abstracts erreicht hat. Ende Jänner 2019 tagte das Programmkomitee des Kongresses in Wien, traf eine Auswahl der besten Beiträge und ordnete die Beiträge den Kategorien Oral presentation und Poster presentation zu.

Neben den eigeladenen Keynotes (7) und State of the art seminars (21) wurden 80 Symposien mit den eingereichten Beiträgen zusammengestellt. Das Programm ist sehr vielfältig und der Besuch der Tagung wird Ihnen Freude machen.

Besondere Freude macht mir, dass es an diesem ESCAP-Kongress erstmals gelungen ist, bei den Hauptrednern ein ausgeglichenes Geschlechterverhältnis zu erreichen.

Univ.-Prof. Dr. A. Karwautz ( $\bowtie)$ Universitätsklinik für Kinder- und Jugendpsychiatrie, Medizinische Universität Wien, Währinger Gürtel 18-20, 1090 Wien, Österreich andreas.karwautz@meduniwien.ac.at
Lesen Sie bitte als Vorgeschmack auf den Kongress die laufend publizierten Interviews mit den Hauptvortragenden (dzt. Prof. Fegert, Prof. Hinney, Prof. Pluess, Prof. Resch, u.a.) unter: www. escap-congress.org und www.escap. eu.

\section{Spezialisierung Psychosomatik}

Um den Bereich Psychosomatik abzusichern, ist es notwendig, dass sowohl FachärztInnen um die Spezialisierung Psychosomatik bei der Österreichischen Ärztekammer ansuchen als auch Abteilungen sich als Ausbildungsstätten für diese Spezialisierung bewerben.

\section{Anträge für Spezialisierung Fachspezifische Psychosomatik für einzelne FachärztInnen}

Der Wunsch um Anerkennung ist mit einem formlosen E-Mail mit Namen und ÖÄK-Arztnummer an die Österreichische Ärztekammer zu senden. Die Ansprechpartnerinnen in der ÖÄK sind: Frau Barbara Janits, MA:

b.janits@aerztekammer.at oder

Frau Nicole Gottsbachner:

n.gottsbachner@aerztekammer.at Abfrage ÖÄK Arztnummer:

https://abfrage.aerztekammer.at/index. jsf
Mit kollegialen Grüßen,

Für den Vorstand

\section{Andreas Karwautz}

ESCAP-Congress President 